\title{
Colestasis intrahepática del embarazo
}

\author{
Intrahepatic cholestasis in pregnancy
}

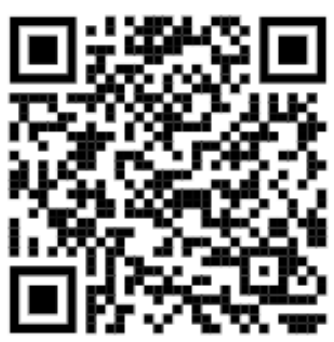

1 Médico general, graduada de la Universidad de de Costa Rica (UCR),código médico: 15174

a.espinozaartavia@gmail.com

${ }^{2}$ Médico general, graduada de la Universidad de Costa Rica (UCR), código médico: 15199. mari v31@hotmail.com

${ }^{3}$ Médico general, graduada de la Universidad de Costa Rica (UCR), código médico: 151200. kembly.webb@ucr.ac.cr

\author{
'Dra. Andrea Espinoza Artavia \\ Investigadora independiente, San José, Costa Rica \\ (D) https://orcid.org/0000-0001-5512-2177 \\ ${ }^{2}$ Dra. Mariana Vílchez León \\ Investigadora independiente, San José, Costa Rica \\ (D) https://orcid.org/0000-0001-7880-869X \\ ${ }^{3}$ Dra. Kembly Webb Webb \\ Investigadora independiente, San José, Costa Rica \\ (D) https://orcid.org/0000-0002-6444-4546
}

RECIBIDO

CORREGIDO

ACEPTADO

$18 / 03 / 2019$

$03 / 04 / 2019$

$26 / 04 / 2019$

\section{RESUMEN}

La colestasis intrahepática del embarazo es la enfermedad hepática específica del embarazo más común, y se presenta típicamente durante el segundo trimestre tardío o tercer trimestre del embarazo. Tiene una etiología multifactorial, que dependerá de características genéticas e influencias hormonales entre otras variables. Se caracteriza por prurito y alteraciones a nivel de parámetros de laboratorio, tales como elevación de ácidos biliares y alteración de pruebas de función hepática. Aún es poco comprendida, y debido a que no se han estandarizado las guías, cuenta con distintos criterios diagnósticos. El pilar del tratamiento consiste en el ácido ursodesoxicólico. Suele ser benigna para la mujer con una rápida resolución posterior al parto, pero está asociada a complicaciones adversas perinatales, entre ellas el parto pretérmino y la muerte fetal intrauterina. Aún queda mucho por explorar y determinar respecto a esta patología de carácter complejo, para lograr alcanzar un consenso entre las distintas guías y conseguir las mejores recomendaciones para la población a escala mundial.

PALABRAS CLAVE: embarazo; colestasis intrahepática; ácidos y sales biliares; ácido ursodesoxicólico; mortalidad fetal.

\section{ABSTRACT}

Intrahepatic cholestasis of pregnancy is the most common pregnancyspecific liver disease and typically occurs during the second trimester or third trimester of pregnancy. It has a multifactorial etiology, which will depend on genetic characteristics and hormonal influences, among 
other variables. It is characterized by pruritus and alterations of laboratory parameters, such as elevation of bile acids and alteration of liver function tests. It is still poorly understood and because the guidelines have not yet been standardized, it has different diagnostic criteria. The treatment pillar consists of ursodeoxycholic acid. It is usually benign for women with rapid resolution after childbirth, but is associated with adverse perinatal complications, including preterm birth and intrauterine fetal death. There is still much to explore and determine regarding this complex pathology, in order to reach a consensus among the different guides and to obtain the best recommendations for the population worldwide.

KEYWORDS: pregnancy; cholestasis, intrahepatic; bile acids and salts; ursodeoxycholic acid; fetal mortality.

\section{INTRODUCCIÓN}

La colestasis intrahepática del embarazo $(\mathrm{CIE})$ es la enfermedad hepática reversible específica del embarazo más común. Se da durante el segundo o tercer trimestre del embarazo, y se caracteriza por prurito con elevación de ácidos biliares y elevación de enzimas hepáticas, en ausencia de otras enfermedades sistémicas o hepáticas (15). Su etiología es multifactorial, y las incidencias varían a nivel geográfico y étnico. Suele ser relativamente benigna para la madre, pero tiene muchos efectos negativos a nivel del feto, incluyendo muerte fetal intrauterina y nacimiento prematuro $(2,4)$.

Pese a múltiples estudios, aún hay opiniones divergentes entre los expertos respecto a los criterios diagnósticos y manejo tanto materno como fetal. Se pretende desarrollar la presente revisión, con vistas a describir el manejo que se tiene actualmente en la colestasis intrahepática del embarazo, así como identificar las posibles brechas que aún existen para futuras investigaciones respecto al tema.

\section{MATERIALES Y MÉTODOS}

Para la elaboración de esta revisión, se buscó bibliografía de los últimos 5 años en Pubmed con los términos "colestasis", "intrahepática" y "embarazo". La búsqueda se enfocó en asociar dichos términos. Fueron relevantes para el objetivo de la revisión aquellos artículos que dieran un especial énfasis al manejo actual de la colestasis intrahepática en el embarazo. Se tomó en cuenta el uso de bibliografía tanto en inglés como en español.

\section{EPIDEMIOLOGÍA}

A nivel global, la CIE tiene una incidencia de $0,2-2 \%$ entre todos los embarazos, mostrando variaciones geográficas, étnicas y estacionales (3). Dicha incidencia suele ser mayor en Sudamérica y el norte de Europa, con una predominancia en países latinoamericanos, siendo Chile uno de los más perjudicados (6). Uno de los grupos étnicos más frecuentemente afectado son los indígenas araucanos de Chile, debido a una serie de alteraciones genéticas en transportadores 
hepatobiliares (7). Otros datos muestran como la incidencia en Estados Unidos es de 2 por cada 10,000 embarazos y en Europa de 20 por 10,000 (7).

\section{ETIOLOGÍA}

La etiología es multifactorial, influyendo tanto factores hormonales como genéticos en individuos predispuestos (2). La hiperestrogenemia que ocurre durante la gestación, particularmente durante el tercer trimestre de embarazo, presenta efectos colestásicos debido a que el estradiol, mediante la activación del receptor de estrógenos, produce una regulación negativa sobre la bomba exportadora de sales biliares "BSEP" (0 bien, ABCB11). Otro mecanismo descrito es mediante el metabolismo anormal de la progesterona, debido a los efectos colestásicos que producen los metabolitos de la progesterona sulfatada al antagonizar el receptor farsenoide $X$ (FXR) y alterar la entrada y salida de ácidos biliares del hepatocito. Estos metabolitos se han asociado con la severidad del cuadro clínico $(2,7,8)$. Asimismo, en aproximadamente $15 \%$ de los casos, el síndrome se encuentra relacionado a una alteración en la expresión de ciertos genes de transportadores de proteínas hepatobiliares, lo que explica el desarrollo de diferencias étnicas y predisposición en familiares de primer grado $(1,2)$. Mutaciones a nivel de las proteínas hepatobiliares transportadoras, tales como el transportador de fosfolípidos acoplado a ATP MDR3/ABCB4, el transportador de amino-fosfolípidos ATP8B1/FIC1, y la bomba exportadora de sales biliares
BSEP/ABCB11, se han visto implicadas en formas familiares de CIE (1).

Adicionalmente, algunos estudios poblacionales han demostrado ciertos factores ambientales que contribuyen al desarrollo de esta patología, tales como la dieta baja en selenio (que se encuentra en comidas como el trigo, pollo, carne y pescado), el consumo disminuido de vitamina $D$, y la época de invierno $(2,6)$.

\section{FACTORES DE RIESGO}

Entre los factores de riesgo se encuentran los antecedentes heredofamiliares o personales de CIE, la reproducción asistida, los embarazos múltiples y la edad materna avanzada (2, 7, 9). Se estima que los antecedentes personales o familiares de CIE tienen un riesgo de recurrencia de entre el 40 al $92 \%$ (1).

Algunas guías, entre ellas la propuesta por el Colegio Americano de Gastroenterología (ACG por sus siglas en inglés), mencionan varias enfermedades hepáticas como factores de riesgo para el desarrollo de esta patología. Entre ellas se citan la colelitiasis y las enfermedades hepáticas virales (1). Ante ello se recomienda el tamizaje con pruebas de laboratorio y ultrasonido para futuro beneficio en estas pacientes (8).

\section{CLÍNICA}

El principal síntoma consiste en prurito no asociado a rash. Típicamente el prurito es más intenso a nivel de palmas y plantas de los pies, de predominio nocturno, el cual suele comenzar durante 
el tercer trimestre de embarazo. Puede ser localizado o generalizado y en casos severos puede haber excoriación por rascado; mostrando en estos casos en el que llega a ser tan intenso, angustia psicológica y alteraciones del sueño. Otros manifestaciones clínicas que la paciente puede llegar a experimentar son: ictericia, anorexia, nauseas, vómitos y esteatorrea debido a malabsorción de grasas $(1,2,7,8)$.

\section{DIAGNÓSTICO}

Las pacientes pueden presentar niveles elevados de ácidos biliares totales en suero, particularmente de ácidos biliares conjugados y predominantemente del ácido cólico. Esta es la alteración de laboratorio más común, reportándose en hasta $90 \%$ de los casos $(4,7,10)$. Igualmente suele presentarse hiperbilirrubinemia leve de predominio directo en hasta $25 \%$ de los casos, y $10 \%$ de los pacientes pueden desarrollar ictericia. Los niveles séricos de las aminotransferasas suelen estar elevados hasta en un $60 \%$ de las pacientes, mientras que los de fosfatasa alcalina pueden estar ligeramente elevados y los de gama glutamil transpeptidasa pueden estar normales o elevados $(1,7,10)$.

El estudio por imágenes revela que no ocurre alteración a nivel del parénquima o de la anatomía hepática. Por su parte, las biopsias hepáticas en estos casos reportan un tinte bilioso de los hepatocitos y tapones biliares a nivel de los canalículos biliares, sin que se produzca actividad necrótica o inflamatoria; estos hallazgos concuerdan con colestasis (7).

En una revisión realizada por Bicocca y colegas, se compararon criterios diagnósticos entre las distintas guías existentes respecto a la CIE. Para esto, se tomaron en cuenta las guías del ACG, del Colegio Real de Obstetras y Ginecólogos (RCOG por sus siglas en inglés), de la Comunidad en Práctica Maternal y Neonatal del Sur de Australia (SAMNCP por sus siglas en inglés), del Gobierno de Departamento de Salud del Oeste de Australia (GWADOH por sus siglas en inglés), de la Asociación Europea para Estudio del Hígado (EASL por sus siglas en inglés) y de la Sociedad de Medicina Materno-Fetal (SMFM por sus siglas en inglés) (1).

EI GWADOH y el RCOG definen la CIE como prurito asociado al embarazo acompañado de una elevación en las pruebas de función hepática o de la concentración de ácidos biliares, sin otra causa probable, que resuelve posterior al parto. Además, se indica que cualquier valor por encima de lo normal esperado para el embarazo en las transaminasas (AST: $3-33 \mathrm{u} / \mathrm{L}$ y ALT: 2-33 u/L dependiendo del trimestre), gamaglutamil-transpeptidasa $\quad(2-26 \quad \mathrm{u} / \mathrm{L}$ dependiendo del trimestre), 0 ácidos biliares (hasta $11.3 \mu \mathrm{mol} / \mathrm{L}$ en el tercer trimestre), puede ser utilizado para fines diagnósticos, y la RCOG especifica que la elevación de los ácidos biliares no se requiere para realizar el diagnóstico $(1,11)$. El ACG, la EASL y la SMFM mencionan que el diagnóstico se da con prurito persistente que resuelve posterior al parto, y valores de ácidos biliares $>10$ micromol/L (1).

La SAMNCP es la única guía cuyo enfoque divide las elevaciones de los valores de ácidos biliares en leves (10-40 $\mu \mathrm{mol} / \mathrm{L})$ y severas (>40 $\mu \mathrm{mol} / \mathrm{L})$. El diagnóstico es sugestivo con prurito que presenta niveles mayores a $10 \mu \mathrm{mol} / \mathrm{L}$, y 
definitivo cuando los valores son $>15$ $\mu \mathrm{mol} / \mathrm{L}$. Además, no incluye los niveles de transaminasas y de gama-glutamil transpeptidasa dentro de los criterios diagnósticos (1).

Todas las guías indican que en caso de que persistan las anormalidades en parámetros de laboratorio posterior al parto, se debe reconsiderar el diagnóstico de CIE. El diagnóstico diferencial de la CIE incluye: preeclampsia, hemólisis, síndrome HELLP, hígado graso agudo del embarazo, abuso de drogas y otras entidades hepáticas o dermatológicas. Otras pruebas de laboratorio para excluir otras etiologías se deberán de valorar según los factores de riesgo individuales (1).

\section{TRATAMIENTO FARMACOLÓGICO Y NO FARMACOLÓGICO}

La terapia de primera línea recomendada por las guías es el ácido ursodesoxicólico (UDCA), un ácido biliar hidrofílico que estimula la síntesis e inserción de transportadores de ácidos biliares en la membrana de los hepatocitos, con lo cual se normalizan las alteraciones en laboratorios, y disminuye el prurito al estimular la excreción de ácidos biliares.

EI UDCA también reduce las concentraciones de ácidos biliares en el feto $y$ protege los colangiocitos $y$ hepatocitos de la madre de lesión celular y apoptosis $(2,6,7)$. Aún no se comprende bien la relación del UDCA con los resultados en eventos adversos perinatales. El estudio PITCHES actualmente estudia esta relación contra placebo en una gran muestra de mujeres de gran Bretaña (2).
Se puede iniciar el tratamiento ya sea de forma empírica cuando los síntomas son evidentes y se descartan otras etiologías posibles, o se pueden repetir las pruebas de laboratorio semanalmente hasta demostrar alteración en los parámetros esperados para iniciar la terapia (10). La ACG y GWADOH recomiendan iniciar con una dosis de UDCA de 10-15 $\mathrm{mg} / \mathrm{kg} /$ día. La SAMNCP por su lado, recomienda iniciar la terapia con $250 \mathrm{mg}$ tres veces al día para CIE leve, y 500mg tres veces al día para enfermedad severa, con un máximo de $750 \mathrm{mg}$ tres a cuatro veces por día $(1,12)$. El UDCA presenta buena tolerancia oral en embarazadas, con diarrea y náuseas como los principales efectos adversos (6). La mejoría sintomática suele darse en aproximadamente una a dos semanas posteriores al inicio de la terapia, y la mejoría en parámetros de laboratorio en tres a cuatro semanas. En caso de no haber mejoría sintomática para las dos semanas, se puede titular la dosis de UDCA hasta un máximo de $21 \mathrm{mg} / \mathrm{kg} /$ día (10).

La rifampicina es una droga de segunda línea que ha mostrado disminución de alteraciones de laboratorio y prurito, usado en combinación con UDCA; mostrando una reducción de estos parámetros en aproximadamente $50 \%$ de las mujeres $(2,8)$.

Las distintas guías no recomiendan el uso de dexametasona como tratamiento, debido a que no tiene beneficios sobre el UDCA y presenta efectos adversos neurológicos para el feto en desarrollo (1). La ACG recomienda el uso de dexametasona únicamente para maduración pulmonar (12). 
Otros tratamientos que pueden mejorar los síntomas y/o disminuir los niveles de ácidos biliares son: la hidroxicina, el fenobarbital, la colestiramina y la Sadenosil metionina (7). Sin embargo, aún no cuentan con suficiente evidencia científica respaldada en ensayos clínicos controlados y aleatorizados; a lo que se suma que en varias ocasiones estas opciones terapéuticas han probado ser inferiores en comparación al UDCA, en cuanto a los beneficios mostrados (8).

Algunos de los tratamientos no farmacológicos descritos son los baños frescos, los baños con avena o con bicarbonato, y las cremas o lociones con mentol. Estos pueden aliviar los síntomas de prurito y causar comodidad (6).

\section{MANEJO DEL PARTO Y COMPLICACIONES RELACIONADAS}

En el embarazo normal, los ácidos biliares están presentes en bajos niveles en la circulación fetal, y se desplazan desde feto hasta la madre mediante la circulación placentaria. En la CIE el gradiente se revierte debido a la elevación de las concentraciones de ácidos biliares en la sangre materna. Niveles superiores a $40 \mu \mathrm{mol} / \mathrm{L}$ en la madre aumentan el riesgo de que el feto presente eventos adversos (2).

La CIE aumenta el riesgo de eventos adversos perinatales severos, tales como la meconización del líquido amniótico e íleo meconial, hipoxia o sufrimiento fetal, partos pretérmino espontáneos 0 iatrogénicos, y muerte fetal intrauterina $(2,3,7)$. El aumento en el riesgo de embarazos prematuros ocurre debido a la estimulación de los receptores de oxitocina, lo que genera contracciones a nivel de miometrio. Los ácidos biliares pueden también alterar el sistema cardiovascular fetal al acumularse en los miocitos, causando trastornos del ritmo en el feto. Asimismo, al acumularse en pulmón puede causar alteraciones en la producción de surfactante, causando atelectasias y dificultad respiratoria en el feto (2). El mecanismo mediante el cual se produce la muerte intrauterina aún no ha sido totalmente elucidado, aunque se ha atribuido cierto nivel de afectación placentaria (8).

La SAMNCP recomienda hospitalizar a las pacientes con valores de ácidos biliares superiores a $40 \mu \mathrm{mol} / \mathrm{L} \quad 0$ ALT>200 IU/L, con toma de laboratorios control dos veces por semana y pruebas de coagulación al momento del diagnóstico de la CIE severa y nuevamente previo al parto. En caso de que se controlen los niveles de ácidos biliares y/o la $A L T$, se puede manejar a la paciente de forma ambulatoria con laboratorios control semanales (1).

El monitoreo fetal anteparto no ha probado ser efectivo en varios estudios $y$ se le ha dado un mal uso, puesto que ha provocado variaciones marcadas al decidir el momento del parto por intentar balancear el riesgo de muerte fetal intrauterina contra los riesgos de un parto pretérmino o de término temprano. Este problema nace a raíz de una falta de consenso entre las guías a nivel mundial; al respecto, las guías publicadas en el 2011 por el RCOG, sugieren discutir con las pacientes la posibilidad de un parto de término temprano como método para minimizar el riesgo de muerte fetal intrauterina, lo cual se respalda por la escasa evidencia científica existente (13). Por lo general, las guías recomiendan un parto entre las 37 y 38 
semanas (1).Pese a que aún no se conoce una terapia efectiva contra la muerte fetal intrauterina, Ovadia y colegas realizaron un meta-análisis de 27 estudios publicado este mismo año, en el cual deseaban determinar la relación entre los marcadores bioquímicos con la posibilidad de eventos adversos perinatales. En dicho estudio, se determinó que las concentraciones de ácidos biliares totales elevadas eran altamente predictivas de muerte fetal intrauterina en embarazos únicos. Según el estudio, el aumento de casos de muerte fetal intrauterina sólo excedía el número de casos respecto a la población normal al alcanzar niveles de ácidos biliares totales de $100 \mu \mathrm{mol} / \mathrm{L}$ o más (14). En dichas mujeres, se recomienda realizar el parto a las 35-36 semanas de gestación. Dado que esto comprende la minoría de los casos, se espera que haya una reducción de partos pretérmino iatrogénicos. No obstante, se recomienda realizar una medición semanal de los niveles de ácidos biliares, debido al aumento que presentan al avanzar el embarazo (13). Las guías también recomiendan realizar pruebas de función hepática semanalmente antes del parto, con un control de pruebas de coagulación en caso de alteración en los parámetros anteriores. Además, tanto el GWADOH como el RCOG recomiendan monitorizar la frecuencia cardiaca fetal durante el parto (1).

Un estudio de cohorte realizado por Liu y colegas que comprendía 1,319 casos de colestasis intrahepática del embarazo, los 163 casos de pacientes con niveles de ácidos biliares entre $40-99 \mu \mathrm{mol} / \mathrm{L}$ no asociaron un aumento del riesgo de muerte fetal intrauterina. Esto es concordante con el estudio realizado por Ovadia y colegas, en el cual se propone el corte de aumento en el riesgo a los $100 \mu \mathrm{mol} / \mathrm{L}$ (13-15).

El parto suele producir completo y rápido alivio de los síntomas, con resultados maternos favorables sin secuelas a largo plazo (7). Sin embargo, la CIE está asociada a patologías metabólicas en la embarazada, debido a que aumenta la susceptibilidad materna de presentar alteraciones en el metabolismo de la glucosa y de los lípidos. Esto ocurre debido a la unión de los ácidos biliares circulantes en sangre con el receptor nuclear de ácidos biliares FXR y el receptor de ácidos biliares acoplado a proteínas G TGR5. Esto provocará un aumento en la incidencia de diabetes mellitus gestacional, dislipidemia y macrosomía fetal (2). La CIE también presenta una asociación con la mayor incidencia de preeclampsia (4).

Varios estudios han relacionado la CIE con deficiencia de vitamina $\mathrm{K}$ producida por la esteatorrea leve de la colestasis o por la terapia con colestiramina, lo cual produce alteraciones en la coagulación y aumentan el riesgo de hemorragia posparto $(7,8)$. Se pueden monitorizar los tiempos de protrombina durante el embarazo con especial atención cerca del parto, y se puede administrar vitamina $K$ de ser necesaria (7). El RCOG y la SAMNCP recomiendan iniciar con una dosis de vitamina $\mathrm{K}$ de $5-10 \mathrm{mg}$ por día en caso de haber elevación en el tiempo de protrombina. Además, la SAMNCP recomienda un manejo activo del tercer estadio del parto (1).

Otros estudios también han demostrado que las mujeres con historia de CIE tienen un riesgo aumentado de 
desarrollar enfermedades hepatobiliares, cardiovasculares e inmunes a futuro, entre las cuales se citan: diabetes, patología tiroidea, psoriasis, poliartropatías, enfermedad de Crohn, hepatitis crónica, fibrosis y cirrosis hepática, colelitiasis y colangitis $(1,2,7,16)$. GWADOH $Y$ SAMNCP describen que mujeres con formas familiares severas de esta patología, tienen un riesgo aumentado de desarrollar enfermedades hepáticas crónicas posteriormente (1). También se ha descrito mayor riesgo de desarrollar cáncer de hígado o del árbol biliar (8).

\section{SEGUIMIENTO}

Según Bicocca y colegas, el prurito suele resolver en uno a dos días y la ictericia en una semana. A su vez, los niveles de ácidos biliares retornan a sus valores normales en una semana y las pruebas de función hepática entre un mes y seis semanas. La mayoría de guías recomiendan realizar pruebas de función hepática entre las dos y seis semanas posteriores al parto. La SAMNCP es la única guía que menciona la repetición de las pruebas de ácidos biliares para ver si normalizan. En caso de que los laboratorios no vuelvan a la normalidad, se pueden considerar hepatitis $C$, cirrosis biliar primaria y colangitis esclerosante primaria como posibles diagnósticos (1).

La lactancia materna no está contraindicada en el posparto (1). Es importante discutir el riesgo de recurrencia para los próximos embarazos. Por lo general, todas las opciones anticonceptivas se encuentran dentro de una categoría uno de recomendación (no hay restricciones), excepto las opciones que contienen estrógeno que se encuentran en una categoría dos de uso (los beneficios suelen superar los riesgos teóricos 0 comprobados) (17).

\section{CONCLUSIONES}

La presente revisión permitió concluir que la colestasis intrahepática del embarazo es una patología compleja y potencialmente peligrosa para el feto, poco comprendida por el personal sanitario, existiendo aún mucho debate respecto al diagnóstico y correcto manejo de la misma. El hecho de que no sea una patología tan frecuente, aunado a la falta de uniformidad de las guías, complica el manejo. Con el abordaje actual, los resultados de laboratorio suelen atrasar el diagnóstico mucho después de la aparición de los síntomas. Es importante mejorar la recolección de datos para el análisis de esta patología a futuro, realizando estudios clínicos retrospectivos, y proponiendo guías por parte de otras organizaciones internacionales que le den importancia a unificar los distintos lineamientos que se deban seguir para el diagnóstico y tratamiento. Además, estas pacientes se pueden ver beneficiadas de un manejo en conjunto con otros especialistas, como dermatólogos, gastroenterólogos, pediatras y obstetras, con el fin de mejorar los resultados tanto para la madre como para el feto.

A nivel de tratamiento, se pueden explorar otras opciones que actúen a nivel de la circulación enterohepática, o que contrarresten el efecto sobre los receptores implicados en esta patología como el FXR y el TGR5. Cabe destacar además, la importancia de terapias que logren disminuir la incidencia de muerte 
Colestasis intrahepática del embarazo - Dra. Andrea Espinoza Artavia; Dra. Mariana Vílchez León; Dra. Kembly Webb Webb

fetal intrauterina, siendo este el comprendido hasta la actualidad. desenlace más sombrío y poco

\section{REFERENCIAS}

1. Bicocca MJ, Sperling JD, Chauhan SP. Intrahepatic cholestasis of pregnancy: Review of six national and regional guidelines. Eur J Obstet Gynecol Reprod Biol. 2018 Diciembre; 231: 180187.https://doi.org/10.1016/j.ejogrb.2018.10.041

2. Chivers S, Williamson C. Intrahepatic cholestasis of pregnancy. Obstet Gynaecol Reprod Med. 2018 Julio; 28(7): 215-217. https://doi.org/10.1016/j.ogrm.2018.06.003

3. Williamson C, Geenes V. Intrahepatic cholestasis of pregnancy. Obstet Gynecol. 2014 Julio; 124(1): 120133. https://doi.org/10.1097/AOG.0000000000000346

4. Dixon $\mathrm{PH}$, Williamson $\mathrm{C}$. The pathophysiology of intrahepatic cholestasis of pregnancy. Clin Res Hepatol Gastroenterol. 2016 Abril; 40(2): 141-153. https://doi.org/10.1016/j.clinre.2015.12.008

5. Henderson CE, Shah RR, Gottimukkala S, Ferreira KK, Hamaoui A, Mercado R. Primum non nocere: how active management became modus operandi for intrahepatic cholestasis of pregnancy. AmJ Obstet Gynecol. 2014 Setiembre; 211(3): 189-196. https://doi.org/10.1016/i.ajog.2014.03.058

6. Gabzdyl EM, Schlaeger JM. Intrahepatic cholestasis of pregnancy: a critical clinical review. J Perinat Neonatal Nurs. 2015 Enero; 29(1): 41-50. https://doi.org/10.1097/JPN.0000000000000077

7. Cappell MS. Obtetrics Normal and Problem Pregnancies. 7th ed. Philadelphia: Elsevier; c2016 Abril. Hepatic Disorders During Pregnancy; p.1008-1011.

8. Ovadia C, Williamson C. Intrahepatic cholestasis of pregnancy: Recent advances. Clin Dermatol. 2016 Mayo-Junio; 34(3): 327-334. https://doi.org/10.1016/j.clindermatol.2016.02.004

9. Floreani A, Gervasi MT. New Insights on Intrahepatic Cholestasis of Pregnancy. Clin Liver Dis. 2016 Febrero; 20(1): 177-189. https://doi.org/10.1016/j.cld.2015.08.010

10. Lindor KD, Lee $\mathrm{RH}$. Intrahepatic cholestasis of pregnancy [Internet]. Waltham, MA: Uptodate; 2019 [citado 2019 Marzo 16]. Disponible en: https://www.uptodate.com/contents/intrahepatic-cholestasis-ofpregnancy/print

11. Cunningham FG. Normal reference ranges for laboratory values in pregnancy [Internet]. Waltham, MA: Uptodate; 2019 [citado 2019 Abril 03]. Disponible en: https://www.uptodate.com/contents/normalreference-ranges-for-laboratory-values-in-pregnancy/print

12. Tram TT, Ahn J, Reau NS. ACG clinical guideline: liver disease and pregnancy. Am J Gastroenterol. 2016 Febrero; 111(2): 176-194. https://doi.org/10.1038/ajg.2015.430

13. Palmer KR, Xiahoua L, Mol BW. Management of intrahepatic cholestasis in pregnancy. Lancet. 2019 Febrero; 393(10174): 853-854. http://dx.doi.org/10.1016/S0140-6736(18)33186-6

14. Ovadia C, Seed PT, Sklavounos A, Geenes V, Di llio C, Chambers J, et al. Association of adverse perinatal outcomes of intrahepatic cholestasis of pregnancy with biochemical markers: results of aggregate and individual patient data meta-analyses. Lancet . 2019 Marzo; 393(10174): 899909.https://doi.org/10.1016/S0140-6736(18)31877-4

15. Liu X, Landon MB, Chen Y, Cheng W. Perinatal outcomes with intrahepatic cholestasis of pregnancy in twin pregnancies. J Matern Fetal Neonatal Med. 2015 Setiembre; 29(13): 21762181.https://doi.org/10.3109/14767058.2015.1079612

16. Wikström Shemer EA, Stephansson O, Thuresson M, Thorsell M, Ludvigsson JF, Marschall HU. Intrahepatic cholestasis of pregnancy and cancer, immunemediated and cardiovascular diseases: A 
population-based cohort study. J Hepatol. 2015 Agosto; 63(2): 456461.https://doi.org/10.1016/i.jhep.2015.03.010

17. Curtis KM, Tepper NK, Jatlaoui TC, Berry-Bibee E, Horton LG, Zapata LB, et al. US medical eligibility criteria for contraceptive use. 2016 Julio. http://dx.doi.org/10.15585/mmwr.rr6503a1 